\section{El arquitecto y su obra}

\author{
Fernando Riquelme S.
}

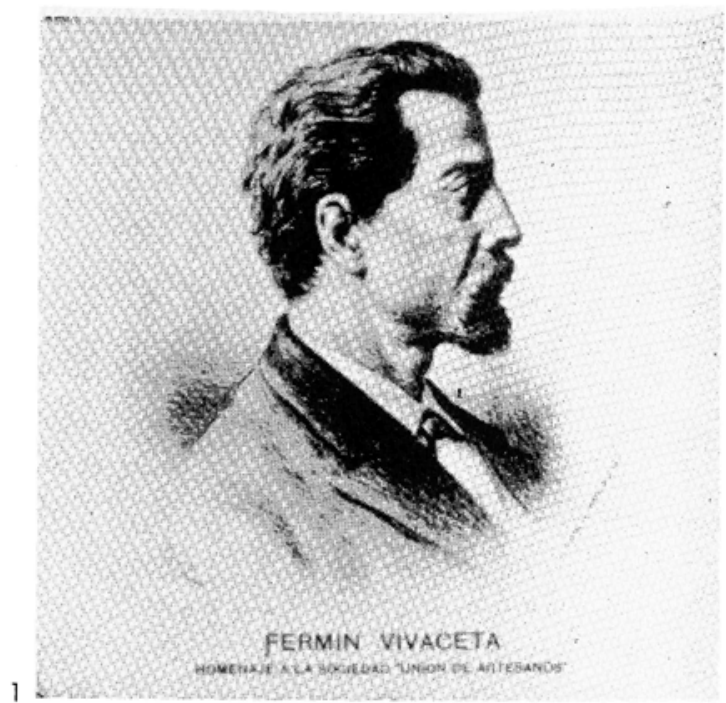

De los arquitectos chilenos formados en los primeros años en que se imparte la enseñanza de la profesión en la Universidad de Chile destacan, a lo menos, tres personalidades interesantes: Manuel Aldunate, Ricardo Brown y Fermín Vivaceta. Aldunate -que terminó sus estudios en Europa- llegó a ser el primer arquitecto chileno de gobierno, cargo que ocuparon sus antecesores franceses, Debaines y $\mathrm{He}$ nault, y es el autor de importantes obras del periodo; el segundo desarrolló una labor muy valiosa, relativamente escasa, en su corta vida y es recordado por su mejor obra, la excelente Galería San Carlos en el costado oriente de la Plaza de Armas de Santiago. Es Vivaceta, sin embargo, el que atrae mayormente la atención por lo multifacético de su labor profesional, artística y social. Efectivamente Fermín Vivaceta va evolucionando, en el transcurso de su vida, desde aprendiz de ebanistería. cuando niño, a dibujante de arquitectura o agrimensor y arquitecto con estudios en la clase de arquitectura, hasta los veinticinco años época en que, acogiéndose a un decreto de gobierno, que autoriza el ejercicio de la profesión a quienes habian cumplido los estudios respectivos, se dedica de lleno a sus labores de arquitectura sin abandonar sus tareas como ebanista y eximio constructor, desarrollando paralelamente una labor social de gran beneficio para la superación de los obreros chilenos.

Gran parte de su vida la dedica al cuidado de su madre, viuda de un militar argentino que él no alcanza a conocer. Su padre que llega a Chile con las fuerzas del general San Martín. había participado en la batalla de Chacabuco. Fermín Vivaceta nace en Santiago en una casa de propiedad de las monjas agustinas, en la primera cuadra de la calle de Ahumada un 12 de Enero de 1829.

Es un estudiante aventajado y debe su formación superior al sacrificio de su madre que, pese a su modesta profesión de lavandera se esfuerza en darle la oportunidad de mejorar sus conocimientos en cursos vespertinos que le van aproximando a su tarea futura como arquitecto:" A la edad de trece años me colocó de aprendiz en un taller de ebanistería: pero conociendolainsuficienteinstrucción que en aquel tiempo se daba en el oficio que yo principiaba a ejercer, tuvo a bien dedicar todo el dinero que su hijo ganaba para pago de profesor, $i$ que continuase en lecciones nocturnas a aprender lo necesario para tener una regular educación" (1). En esos cursos yen los universitarios, que continuara posteriormente, recibe distinciones y premios que le van ga- nando el crédito de algunas personalidades influyentes de la sociedad de la época: "Conservo aún tres diplomas de primeros premios obtenidos en mis exámenes. Conservo. además una medalla de plata que obtuve por algunas láminas de dibujo lineal y ornamental, para servir de modelo, conforme a lo prescrito en el decreto supremo por el cual se me acordó tal distinción" (2).

En esta época de su formación el propio Vivaceta reconocía la positiva influencia de su maestro José Zegers en la clase de Dibujo Lineal y Ornamental para Artesanos del Instituto Nacional; "Su método como profesor, sus conocimientos aventajados en toda clase de dibujos, su inteligencia en las matemáticas, las nociones del arte de edificar, acompañadas de los órdenes de arquitectura, que nos daba, me llamaron la atención, y me indujeron a cambiar mi oficio de ebanista por el de constructor de edificios". (3)

No es de extrañar, entonces, que antelareducida o casi nula existencia de profesionales idóneos en los años previos a la llegada de los maestros franceses contratados por el gobierno, se hubiese entregado al joven Vivaceta, de sólo escasos dieciocho años de edad, el diseño y la construcción de la Casa de Orates con el aval de don José Tomás Urmeneta poderoso industrial y connotado hombre público que le conocía y le apoyaba quizas por su común raigambre chileno-vasca. No podemos asegurar si el plano del edificio mencionado corresponde al publicado años después, con motivo de la Exposición Universal de París en 1889, por el Dr. Adolfo Murillo en "Hygiene et Assistance Publique au Chili" para la Sección chilena en el Pabellón París, pero no hay duda que representa la planta elaborada por una persona sin mayor experiencia dada su falta absoluta de modulación y de órden. (Fig. 1).

En 1853 cuando ya cursa el segundo año de arquitectura recibe un segundo premio consistente en la obra de John Millington, en dos tomos, en que sobre la firma del Rector don Andrés Bello y del Secretario de la Facultad Ignacio Domeyko se lee: "Universidad de Chile, año 1852. Segundo premio de la Clase de Arquitectura. (4)

Dado al alumno Don Fermín Vibaceta(Sic)"

Ensu labor profesional influyeron poderosamente sus maestros, los arquitectos franceses Debaines y Henault. Siente por ellos gran respeto, asi lo manifiesta cuando a solicitud de su biógrafo, José Bernardo Súarez da su opinión sobre el primero: "Losedificios del Señor Debaines, dice el señor Vivaceta, se distinguen por una remarcable sencillez, i a juicio de muchos, son inferiores a los que hoy se construyen mezclados de diferentes estilos i caprichosas proporciones; pero el tiempo hará justicia al mérito del arte $i$ entonces mejor que ahora se conocerá que el arquitecto de aquellas obras poseía profundos conocimientos del arte, $i$ que inspirado por la severidad del estilo griego i romano en su pura forma i proporciones quiso dejarnos ese tipo de construcciones, como un testimonio de su viaje $i$ detenidos estudiosen losmonumentos de Grecia iRoma" (5)

Brunet Debaines debió transmitir a Vivaceta el gusto por la sobriedad del lenguaje formal del renacimiento, que encontraba representado en la obras griegas y romanas de la antiguedad y que se habían traducido en los grandes tratados de arquitectura de los maestros del renacimiento en ideas muy claras y definidas sobre los órdenes clásicos, la forma y la proporción de los edificios. Confirma esta inclinación hacia lo clásico, en esta nueva visión de mediados de siglo en Debaines y en Vivaceta, la abundante bibliografía que este último posee de los tratadistas del renacimiento, en castellano, en pulcras ediciones. En la contratapa de uno de ellos hemos podido leer recientemente, manuscrito, a lápiz y con su rúbrica; "En 1852 compré este libro en 24 pesos", es una hermosa edición del "Tercero y Quarto libro de Architectura de Sebastián Serlio. Boloñez, traduzido de Tofcano en lengua castellana, por Francisco de Villalpando, Architecto. Conlicencia impreffo en Toledo, Año de 1563, con PRIVILEGIO". Había adquirido una de las obras que mayor influencia tuvo en la arquitectura, verdadera joya del pensamiento renacentista; las ideas de Serlio ysus grabados, realizados con precisión y esmero debieron dejar profunda huella en la mente fresca y ávida de perfeccionamiento de Vivaceta.

Poseía una edición del Vitruvio de "Los Diez libros de Arquitectura, de M. Vitruvio Polion, Año 1787" en versión castellana de Dn Josef Francisco Ortiz y Sanz, Presbítero y también en versión castellana "traducidose ilustrados con notas" del mismo Ortízy Sanz "Los Quatro Libros de Arquitectura de Andrés Paladio, Vicentino" Año de 1797. "Madrid en la Imprenta Real siendo rejente Dn. Pedro Julian Pereyra, Impresor de Cámara de S.M.".

Había obtenido, además, al parecer como Primer Premio de las Artes del Instituto Nacional de Chile en 1850, "Los Diez Libros de Arquitectura de León Baptista Alberto" en segunda edición en castellano corregida por D.R.B. en Madrid, 1797. Un timbre, del propio Vivaceta, 


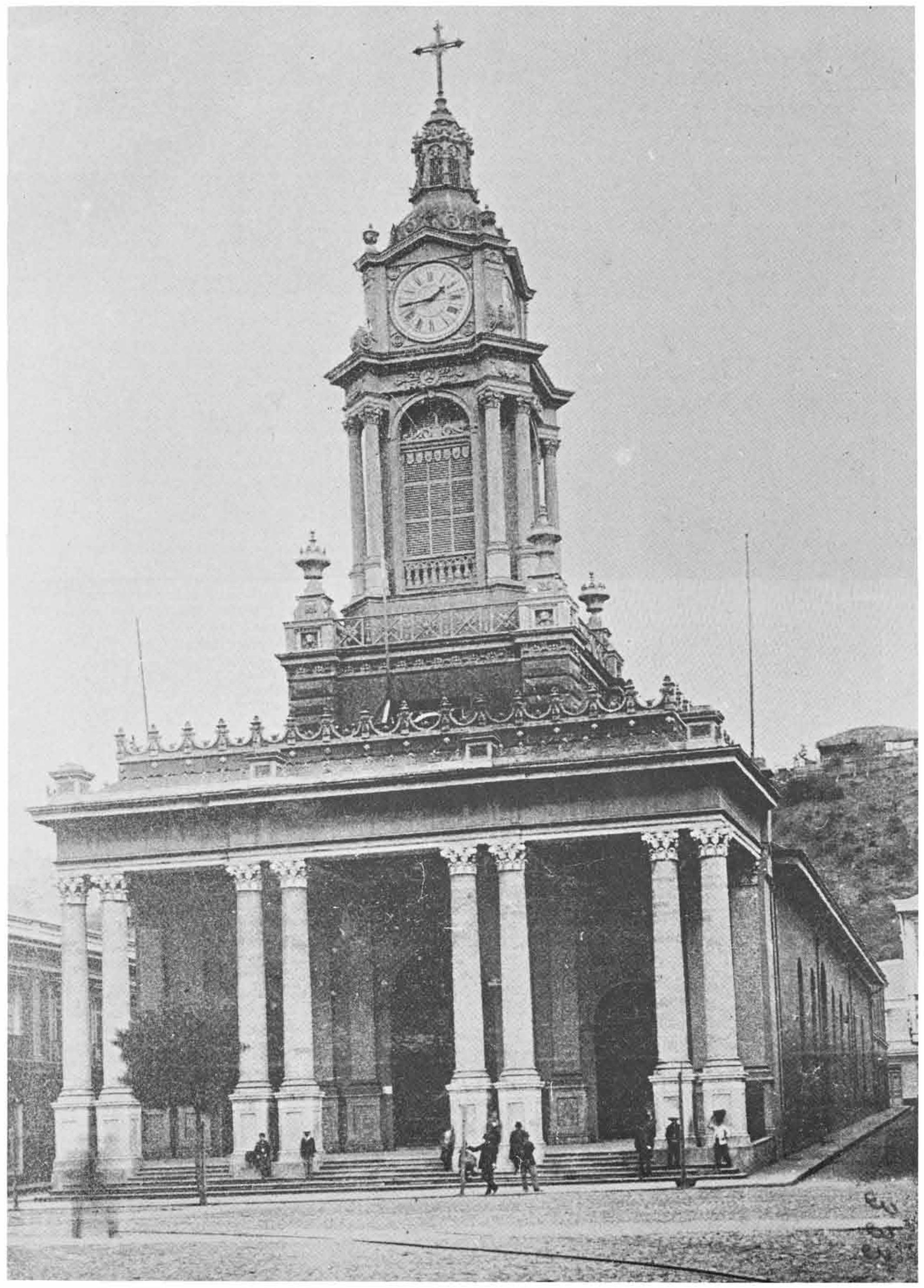



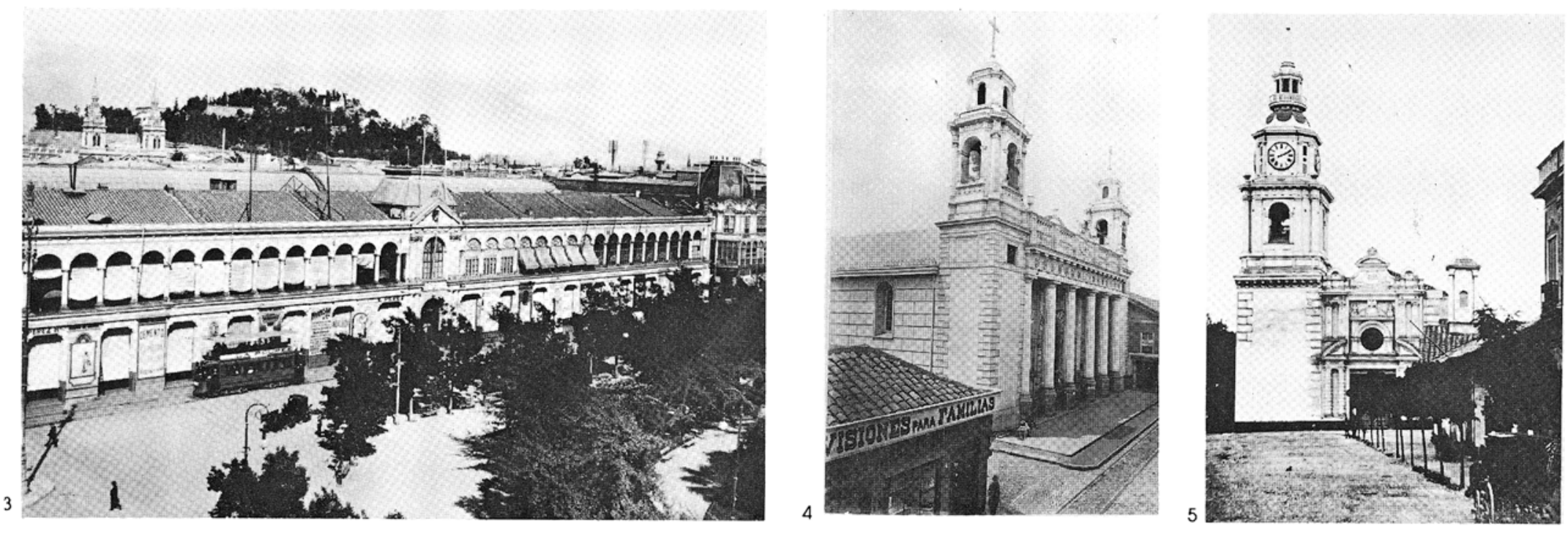

certifica la calidad del premio, en la primera página.

Si el conocimiento que tuvo Vivaceta del Tratado de Alberti, a los veintiún años, pudo ser fundamental en su formación teórica los tratados de Serlio, de Vitruvio y de Palladio tienen que haberle afianzado en losideales renacentistas y neoclásicos. Si a ello se añaden las excelentes obras de matemáticas y geome tría descriptiva que formaban parte de su biblioteca como un "Curso de Matemáticas para uso del Ingeniero Civil" de J. Adhemar, 1844 - el "Album práctico del Arte Industrial" de C.A. Oppermann o la "Tabla Alfabética y Analítica de Materiales", París 1864, y la "Architecture Moderne ou L'Art de Bien Batir" de Ch. A Jombert, 1764 y quizás cuantos otros que no hemos encontrado, no nos cabe duda sobre la sólida base de formación profesional que Vivaceta se había labrado con su clara vocación y espíritu de superación. Esa vocación hacia la arquitectura que impulsó a Zegers, su profesor en el Instituto, a enseñarle "geometría descriptiva, trigonometríayseccionescónicas".

Esta verdadera colección de tratadístas clásicos y renacentistas convierten a Vivaceta en un Arquitecto de cultura superior y explica su capacidad para desenvolverse con soltura y señorio frente a los desafios que le demanda su tarea profesional. No es menos, importante. sin embargo, el trato que tiene por largos años -más de dos decadas- con los arquitectos franceses contratados como arquitectos de gobierno desde 1849 a 1872 año en que el segundo de ellos Luciano Ambrosio Henault decide volver a su país de origen. Por aquellos años se habían incorporado, además al quehacer arquitectónico nacional importantes figuras europeas como el italiano Eusebio Chelli, romanoy Teodoro Burchard, alemán. Conellos participó Vivaceta en diversas obras en Santiago y en Valparaíso. Pero es, sin duda con Debaines y Henault con quienes desarrolla laboresen común de mayor relevancia. Asi para Brunet Debaines diseña el frontis del edificio inconcluso del portal Tagle en el costado oriente de la Plaza de Armas con una expresión marcadamente renacentista, a la manera de Brunelleschi; para él también completa y construye la casa de la familia Mac Clure en la acera oriente de la calle Bandera esquina de Compañía, enfrentando al edificio del Congreso Nacional y que se mantuvo en pie hasta la década del 60 , de fachadas muy sobrias, de líneas armónicas por sus acertadas proporciones, propias del estio neo clásico, más justamente, neo renacentista.

Cuando Brunet Debaines fallece, la vispera de su partida de regreso a Francia, Vivaceta debe asumir la construcción de algunas importantes obras iniciadas por el arquitecto francés, entre ellas el Templo de la Veracruzen la calle Lastarria, en la que ya dirigía su construcción, además del portal Tagle o Mac Clure, en la Plaza de Armas.

Tanto o más importante es la responsabilidad que asume Vivaceta en su colaboración con Henault, el segundo arquitecto de gobierno. Conél construye el edificio llamado entonces, Palacio de la Universidad de Chile, actua casa central universitaria y sede de la rectoría. Cuando Henault regresa a su país asume la dirección total de la obra, introduciendo modificaciones al proyecto original del francés y actuando, entonces, como arquitecto y contratista de la obra. Para esa fecha Vivaceta ya se había ganado el respeto de las autoridades universitarias, de personalidades de gobierno y de sectores respetables de la sociedad santiaguina. Formaba parte desde hacía algún tiempo (aproximadamente 1852) de la Junta Directiva en la Organización de la Sociedad de Instrucción Primaria; "donde desempeñó importantes comisiones que le granjearon el aprecio de sus consocios, los señores Miguel Luis Amunategui, Alvaro Covarrubias, Benjamín Vicuña Mackenna, Domingo Santa María, Joaquín Blest Gana y otros respetables señores que tan valiosos servicios prestaron a la organización de dicha asociación, que ha derramado la luz y el progreso entre los humildes". (6)

Entre otras labores Vivaceta reemplazaba a los profesores que no asistían a sus clases en las Escuelas Nocturnas para adultos y que mantenía dichaSociedad, recorriendo sincansancio las Escuelas "desde el barrio de San Miguel (hoy Yungay) hasta el de Reco!eta". (7).

La iglesia católica, por otra parte le fué confiando trabajos cada vez de mayor envergadura e importancia, llegando a ser arquitecto director de los trabajos que se efectuaron en la Catedral entre 1864 y 1867, año en que se suspendieron a raíz de la guerra con España. El Arzobispo de la Diócesis don Rafael Valentín Valdivieso resolvió su nombramiento por cuanto había demostrado "acierto en obras de este género". Se trataba de obras muy diversas que incluían desde refuerzos estructurales de la torre y de techumbre hasta construcciones interiores muy decoradas, esculturas e incluso muebles. En todas ellas Vivaceta demostraba su dominio de la técnica y de la artesanía ejecutando dorados, tallas e incluso pinturas como estos casos señala- dos en un contrato: "7ㅇ Construir un nuevo tabernáculo haciéndolo de marmol artificial (escayola) según el órden corintio con sus molduras y adornos dorados, todo incluido por \$5.600.-" o el siguiente "8o Por la obra de escultura que cubre el espacio entre la bóveda o techo de la nave central y el remate del tabernáculo, representando la Asunción de Nuestra Señora a los cielos...\$2.000.-" Los trabajos sumaban, en esa ocasión \$14.088.- (8)

El "acierto en obras de este género" lo venía confirmando Fermín Vivaceta desde sus primeras realizaciones religiosas. Siendo muy joven había diseñado y construido, -presentando veinte planos de su proyecto a una comisión examinadora-el púlpito de la iglesia de Santa Ana en Santiago, hoy desaparecido. Su trabajo mereció elogios pero también dificultades en el pago: nombrado un perito tasador, éste manisfestó, "el trabajo que ha ejecutado, es irreprochable, y vale dos veces más que el precio en que lo ha convenido".

Realiza, cuando cuenta con veinte años de edad, el púlpito de la iglesia de San Agustín, por el que recibe \$750.- Es una obra de gran calidad y demuestra la maestría de Vivaceta como tallador y diriamos casi escultor por la gracia y finura en la ejecución de la figura del Santo en el óvalo del fondo.

Son multiples las tareas que cumple en varios templos de Santiago y más adelante en Valparaíso diseñando y realizando a veces de su propia mano obras de tallado y carpintería, como la puerta de la Iglesia de San Agustín; el púlpito de la Iglesia de la Viñita que él obsequia a dicha parroquia o el remozamiento del púlpito de la Basilica de la Merced donde la cuantía de su participación, de la que no cabe duda, parece ser más importante de lo que se supone.

Documentación existente deja claro testimonio de su autoría en el proyecto y la realización de las torres y del frontis de la lglesia de San Agutín, en Santiago, durante los años 1853 al 1855. (Fig.4). Aquíinicia Vivaceta el tema del campanario de las torres, y del pórtico que pocos años después desarrollará en los templos de San Francisco en Santiago y del Espíritu Santo en Valparaíso.

La obra que le ha hecho más popular y a la cual se le reconocen méritos arquitectónicos indudables, es la torre de la Iglesia de San Francisco: su imagen se ha convertido, muchas veces, en símbolo de la ciudad. No ha faltado quien considerándola anacrónica o poco original ha propuesto su desaparición para, de paso "enderezar" la circulación de la vía vehicular que la torre enfrenta con gracia y 

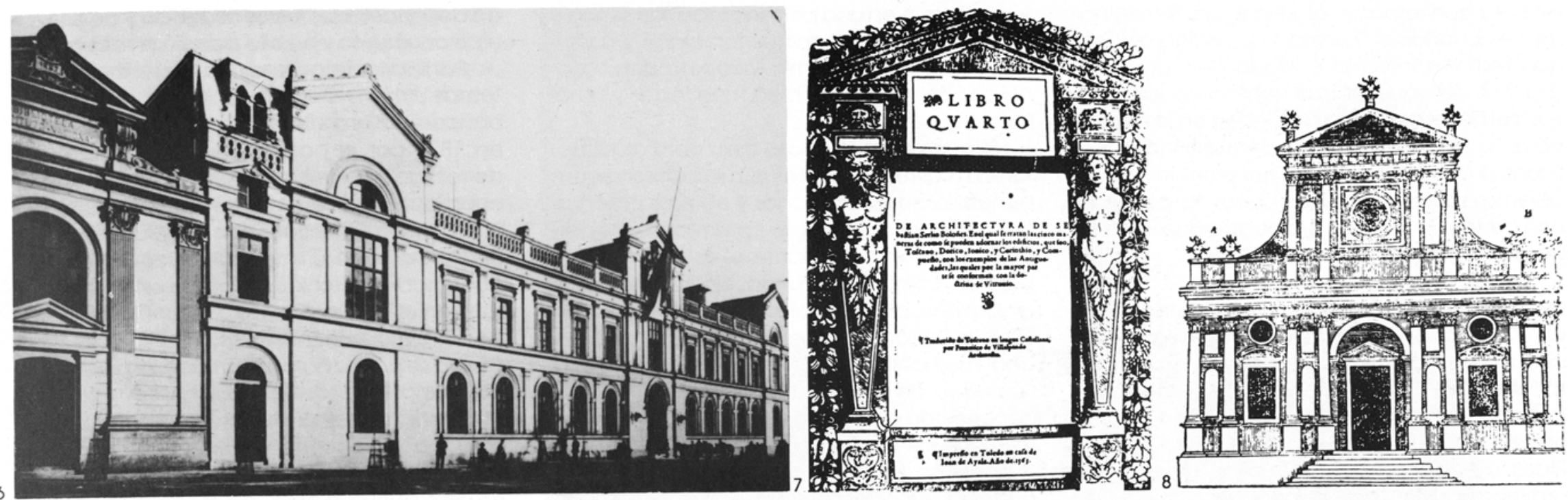

decisión.

Vivaceta enfrentó en esa ocasión, 1858, el desafío que se le propuso de dotar a la iglesia de una nueva torre, con idoneidad y audacia. (Fig. 5).

El templo poseía una torre diseñada, al parecer por un sacerdote de la órden que según podemos apreciar en el grabado que realizó J. Gandarillas en la época, no estaba bien resuelta, era chata y desproporcionada y no tenía una clara estructura formal arquitectónica, ni menos estilo reconocible. El imagina para San Francisco una nueva torre, de gran altura, que debía dominar el paisaje de la ciudad en la que sobresalian sólo algunas. muy pocas entonces, siendo las más destacadas por su fuerza expresiva y su volumen las de la iglesia de Santo Domingo. Cinco años antes él mismo había levantado las dos más menudas de San Agustín, a sólo tres cuadras de San Francisco.

El motivo central del diseño, -un campanario de base cuadrada, ochavado para contener una columna exenta, de órden dórico en cada ochavo- es muy similar en ambos templos. Se diferencian por el remate superior, más complejo y más alto en San Francisco, que da cabida en un segundo tambor menor, también ochavado, de similares características y que contiene un reloj de cuatro esferas orientadas hacia los cuatro puntos cardinales y que "habrá de prestar grandes servicios a la población", según un comentario en la época de su inquguración.

Es interesante constatar la evolución del diseño de las torres de Vivaceta, desde San Agustín a San Francisco en Santiago y veinticinco años más tarde en la Iglesia del Espíritu Santo en Valparíso (1880) en que la misma temática anterior se vuelve más complicada. casi barroca, por el juego de cornisas entrantesy salientes, por el juego de dobles columnas exentas que enmarcan el gran vano central con arco de medio punto, que sirve de bocina del campanario hacia los cuatro vientos de la ciudad. En los tres casos existe una superposición de volúmenes decrecientes en tamaño hacia la altura rematando en un chapitel en que experimenta diferentes soluciones de detalle, cada vez más complejas y dinámicas. que sin embargo no alteran la similitud en el conjunto. Más barroca es la exageración de la altura del dado del campanario en la torre del Espíritu Santo, que nos atrevemos a insinuar. pudo haber servido de inspiración a Eduardo Provasoli el arquitecto italiano que diseña la torre del Templo de San Francisco de Barón en Valparaíso y la del Templo de la Divina Provi- dencia en Santiago algunos años después, de ligero parecido. No así en el pórtico en que Vivaceta demuestra, claramente a nuestro juicio, su inclinación a las formas clásicas por su apego a los tratadístas del renacimiento. Es evidente en este caso la inspiración de Vivaceta en la "fachada para una iglesia" que propone en su tratado Sebastián Serlio y que dibuja con un pórtico formado por tres vanos generados por el juego de pares de columnas corintias que sostienen un entablamiento $o$ arquitrabe de cierta robustez.

La solución del pórtico dado por Vivaceta es apenas diferente a la proposición serliana en la menor distancia del intercolumnio de las dobles columnas en que Serlio incluye un dibujo de figuras simples que Vivaceta suprime. Pero más evidente es el parecido si examinamos el plinto o basamento de las columnas que en ambos casos es casi idéntico y de una altura poco habitual.

Pudo conocer Vivaceta grabados o algún libro con láminas de las obras de Perrault de la fachada oriental del Louvre, solucionada con columnas acopladas, o pudo haber examinado en la misma forma el pórtico de la Catedra de San Pablo en Londres de Wren y tomar de allí la solución del pórtico para el Espíritu Santo no podemos asegurarlo por el momento, pero sí sabemos que debió recorrer muchas veces las páginas del tratado de Serlio o de los cuatro libros de A. Palladio, su contemporáneo, y que de allí supo elegir muy adecuadamente los modelos que le llevaron a dar acertadas soluciones arquitectónicas a los edificios que le tocó diseñar, ampliar o refaccionar.

Esta idea sobre la admiración y el respeto de Vivaceta por Serlio que, como vimos, conoció directamente cuando adquirió el Tratado en su juventud, se refuerza aún más cuando le vemos realizar otra de sus obras importantes en Valparaíso. En el Templo Masónico, construido el año 1872 en la calle de la Victoria, hoy Avenida Pedro Montt, Vivaceta utiliza sendas "Serlianas" para enmarcar el pórtico de acceso a nivel del segundo piso, pórtico que aparece enmarcado, a suvez, por gruesas columnas corintias de órden gigante, solución a la que Palladio recurre frecuentemente en Vicenza y en Venecia. Vivaceta como Palladio encuentran en Serlio -a gran distancia en el tiempo y en el espacio- , una común inspiración. Lo que hace más notable en el chileno su aproximación a los maestros del renacimiento es que ésta se produce en un ambiente carente de medios y de estímulos artístico; sin la vivencia del pasado arquitectónico europeo o extranjero, pués él nunca salió de Chile.
Las opiniones sobre la calidad de este último edificio le fueronmuy favorables. Asi decía J. Bernardo Súarez en el "Plutarco del Artísta Americano" en 1872; "en Valparaíso, la pricipal obra del Señor Vivaceta es el hermoso edificio del club Mazónico (Sic), notable por la inteligente distribución de sus departamentos. belleza y alegría, tenido por una de sus mejores obras". Tornero en el "Chile lustrado", por su parte, destaca el edificio de propiedad de Dn Antonio Subercaseaux en el centro de la ciudad que "se hace notar por su elegante frontis i su magnífico estuque". El mismo Torneo hace una descripción del "grandioso edificio de Dn. Francisco Ossa" de cerca de dos mil metros cuadrados de construcción que Vivaceta diseño y construyó en 1870, también en Valparaíso y que por encontrarse en zona de fuerte pendiente poseía, dos niveles por la calle Prat y tres por la calle Cochrane. Fue hasta hace algunas décadas uno de los ejemplos más destacados de la arquitectura de líneas neoclásicas más rigurosas existentes en nuestro puerto principal. Poseía sendas fachadas simétricas de frontón triangular central por ambas calles. Mientras por la calle Prat el pórtico de acceso lo constituían tres arcos que descansaban en fuertes columnas corintias que alcanzaban los dos pisos de altura: por la calle Cochrane un pórtico de seis columnasen el mismo estilo ponían una nota manierista al no llegar al suelo y quedar interrumpidas por el primer piso. Ambas fachadas siendo diferentes eran singularmente unitarias, Vivaceta jue ga con mucha soltura haciendo una arcada continua de medio punto en el primer nivel y ventanales de marcado dintel recto en el segundo por calle Prat para invertir el sistema totalmente por la calle Cochrane, en que los arcos de medio punto están en el segundo piso. La tercera calle que enfrenta el edificio. Urriola, le permite unir acertadamente ambos sistemas de fachadas.

Sin embargo, es en el espacio interior del gran hall de doble altura donde era posible captar la capacidad de imaginación y fuerza expresiva de la arquitectura de Vivaceta apegada, a los moldes neoclásicos. Los escasos testimonios que restan de dicha obra nos permiten observar como resuelve, nuevamente en el lenguaje de sus maestros renacentistas, un espacio centralizado de gran altura. pero muy bien proporcionando teniendo el círculo, como forma perfecta, para su realización. Serlio y Palladio continúan presentes ene conjunto y en los detalles. Así, es posible encontrar una relación muy directa entre la forma que Vivaceta da a las pilastras en forma de 
estipites que rematan en una figura femenina de tres cuartos o "hermas", y las figuras con que Serlio, adorna la portada del "Libro IV" (Fig. 7) o de aquellas que conforman los apoyos del dintel para una chimenea en la misma obra. Es sabida y ha sido demostrada la influencia que los tratadistas tuvieron en América en los siglos XVI al XVIII (9), pero no sabemos con certaza cuanto se prolonga aquella en el pasado siglo.

Las realizaciones de Vivaceta en el campo de la arquitectura residencial son abundantes en Valparaíso y en Santiago. Curiosamente cuando uno de sus grandes amigos y protector, don José Tomás Urmeneta -"el hombre del cobre"- decide las construcción de su casa habitación, en la calle Monjitas cercana a la Plaza de Armas en Santiago, le entrega el proyecto a Manuel Aldunate, Arquitecto de Gobierno -sucesor de L. Henault en dicho cargo- quien siguiendo con su línea historicista y ecléctica, ya demostrada en su trabajo del llamado Palacio de la Alhambra para otro rico industrial minero, diseña una "enorme estructura de paradojal estilo gótico inglés, siglo XIX" (10). Al parecer los estudios hechos por Aldunate en Europa le habían puesto más al día en las experiencias historicistas, especialmente en las preferencias por los estilos medievales, neogóticos y neorománicos, libremente interpretados y con dudoso gusto. Vivaceta ajeno a las nuevas búsque das de la arquitectura europea se mantenía fiel al legado de sus maestros y a los dictados de sus estimados libros renacentistas vitruvianos, realizando obras de mejor calidad estética.

No se puede afirmar, sin embargo que la arquitectura de Vivaceta no tengan puntos débiles; el mismo Templo Masónico de Valparaíso a pesar de sus raices neoclásicos en Serlio y en Palladio produce, en el conjunto de su fachada, una sensación desfavorable por la intromisión de formas ajenas al rigor del estilo en su remate superior que acusa en él una tendencia a la decoración abundante y exótica de tipo orientalista que vuelve a aparecer en algunos remates superiores del Espíritu Santo o sobre los vanos interiores de puertas que dan a gran hall circular de la casa Ossa. También en Valparaíso y que hemos podido ver en los planos de relevamiento, hechos antes de su demolición. (11).

Hay, también un momento en el desarrollo de su obra en que el movimiento historicista de tendencia neomedieval conquista la simpatía de Vivaceta; en 1865 diseña y construye para las Hermanas Carmelitas el Templo del Carmen Alto en estilo neogótico, al menos en su pórtico y su torre, ya que en las fachadas laterales de la nave se limita a modelar una serie de arcos apuntados en el muro nada transparente, salvo en la puerta de acceso lateral que conforma uno de los arcos ojivales. (Fig. 8).

Su ubicación en plena Alameda de las Delicias esquina de calle Carmen, donde curiosamente hoy día a venido a dar la escultura en bronce del arquitecto, que le rinde justo homenaje, llamaba la atención por su curiosa imagen que constituía una forma exótica y extraña en la morfología de la cuidad. ¿Qué llevó al arquitecto a renunciar a su lenguaje clásico anterior? Pudo ser una exigencia de la congregación o ¿fué una aproximación a los hermanos constructores medievales de la "maçonnerie", por la cual se sentía ya tan atraido y que se simbolizaban fielmente en el racionalismo gótico? No lo sabemos. Pero, sí podemos suponer que en una personalidad tan sensible y de principios humanistas tan claros y definidos como los de Fermín Vivaceta debía corresponder a razones bien meditadas y no al fruto del azar.

Si interesante y valiosa es su obra arquitectónica más respetable es aún su labor social en beneficio de los artesanos y obreros chilenos. Llevando por sus ideas de fraternidad y de perfeccionamiento mediante el estudio y el trabajo en comunidad funda, con un reducido grupo de colaboradores, el 12 de Enero de 1862 la "Unión de Artesanos", primera sociedad mutualista de Chile que hoy se llama Sociedad de Artesanos "La Unión" y que ha beneficiado a varios miles de asociados en su más que centenaria existencia. Pocos meses después en Julio del mismo año, inaugura con asistencia del Presidente de la República don José Joaquín Pérez-a quien le había diseñado y construido su casa- la Escuela Nocturna de Artesanos. En esa ocasión señaló Vivaceta en parte de su discurso: "La fraternidad esla patria de todas las naciones; todos los hombres son hermanos, su culto ysu origen es Dios" y añadía más adelante: "Es muy natural, Excelentísimo señor, que uno de los principales elementos del socorro mutuo lo consideremos los artesanos en el inagotable recurso de propagar la enseñanza, para obtener gradualmente el progreso y con éste el bienestar". (12)

La sociedad llegó a constituir un organismo de indudable trascendencia social y política recibiendo el reconocimiento de connotados hombres públicos entre los que figuran varios presidentes de la República que la visitaron para testimoniar su apoyo a la tarea social humanitaria que representó su creación. Fermín Vivaceta ganó con ello un puesto en la historia social del país, su lugar en la historia del desarollo artístico y cultural, a veces olvidada (13), merecer ser destacada mediante el examen más riguroso y justo de sus obras.

Su afán de servicio le llevó a realizar, todavía. muchas acciones de noble inspiración. El gran incendio del Templo de la Compañía de Jesús en Santiago, en Diciembre de 1863, que lleva a la fundación del cuerpo de Bomberos cuenta a Vivaceta entre sus primeros voluntarios. Con la Tercera Compañía acude al puerto de Valparaíso con ocasión del bombardeo a que lo somete la escuadra española en 1866: crea allí un "cuerpo de voluntarios" para la defensa de la ciudad.

En los momentos críticos de la Guerra del Pacífico trabaja gratuitamente en la dirección de los trabajos de algunos fuertes y cuarteles: forma asimismo un cuerpo de Zapadores obreros destinado a reconstruir rápidamente las defensas dañadas y se encarga de formar un cuerpo de socorro para ayudar en la sepultación de las víctimas de la guerra que fallecían en los hospitales de Valparaíso.

Sus ideas políticas estaban firmemente ligadas a su pensamiento social humanista. Fué admirador de Francisco Bilbao, el creador de la Sociedad de la lgualdad que añoraba repetir en Santiago el levantamiento de París de 1848 -el mismo que motivó a Víctor Hugo su "Napoleón el pequeño"- sin embargo Vivaceta entendía la lucha por las clases desposeídas de muy diferente manera: "La influencia benéfica y democrática de la vida republicana. hace residir la igualdad en la inteligencia y el trabajo", afirmaba, (14). Tenía una fé casi absoluta en la educación y en el trabajo como seguros contra la miseria.

Fué integrante de una comisión encarga- da de repatriar los restos de Bilbao y de erigirle un monumento y en ella trabajó sin descanso.

Partidario de la candidatura del liberal José Tomás Urmeneta a la presidencia de la República en 1870y de Benjamín Vicuña Mackenna en 1875 por el partido liberal democrático. derrotado en ambas, logró el triunfo de su candidato con el Presidente don Domingo Santa María y después con don José Joaquín Pérez. Con éste último, que le conocía bien, pudo haber obtenido un cargo en el Congreso, o en el Municipio pero "Ios rehusó en absoluto: no sin razón, pués creía que por su intermedio se quería halagar artificiosamente a los obreros". (15)

En sus últimos años de vida continúo desplegando una permanente actividad con proyectos que no se realizaron o que se iniciaron en parte y que le califican, además, como un inteligente urbanista. En efecto, se preocupó de financiar personalmente trabajos de nivelación en el Camino de Cintura del puerto y de diseñar poblaciones urbanas para que los obreros pudieran adquirir sus viviendas "librándose de vivir en los conventillos". Para tal efecto fundó la "Asociación de Trabajadores" en 1877, una sociedad cooperativa. Su proyecto, al parecer, proponía, adelantándose a su época, la construcción de una vía ferrea que uniría a media altura el cordón de cerros del sur de Valparaíso y que circularía entre los grupos de edificios mencionados. La Guerra del Pacífico y un grave transtorno de su salud, cuando inspeccionaba las obras del frontis de la Iglesia del Espíritu Santo en 1882, dejaron esos proyectos sólo en el papel.

Para aliviar en parte su difícil situación económica, quiso recurrir a la venta de su excelente biblioteca; el escultor José Miguel Blanco, su amigo propuso crear un fondo para tal efecto entre sus amigos y admiradores. El primero en adherirse fué el Ministro del Interior, don José Manuel Balmaceda y también lo hicieron Miguel Luis Amunategui, Diego Barros Arana, Ignacio Domeyko, Manuel Renjifo y muchos otros connotados intelectuales y políticos de la época. Desarrolló todavía, con dificultades algunas tareas, como ciertas investigaciones de una mezcla o "conglomerado" con el que se proponía reemplazar el uso de la piedra en columnas y pavimentos. El mismo lo había ensayado, con éxito, en las columnas del Espíritu Santo, pero sus experiencias no prosperaron. Falleció poco tiempo después, el 21 de Febrero de 1890 a los 61 años de edad en Valparaíso, en la calle Waddington.

\section{CITAS BIBLIOGRAFICAS}

1. José Bernardo Suárez "Plutarco del Artista Americano", 1872, Santiago de Chile.

2. José Bernardo Suárez. Op. Cit.

3. José Bernardo Suárez. Op. Cit.

4. De la Biblioteca de Fermín Vivaceta en la Sociedad de Artesanos La Unión.

5. José Bernardo Suárez. Op. Cit. pág. 150

6. Arturo Blanco A. "Vida y Obras del Arquitecto Don Fermín Vivaceta" 1924. Santiago de Chile, pág. 17.

7. Arturo Blanco a. Op. Cit. 
8. Archivos del Arzobispado de Santiago. Citado por A. Wood Le Roy "Fermin Vivaceta". Seminario Tesis para optar al Título de Arquitecto, 1956. Inédito, Instituto de Historia de la Arquitectura. Universidad de Chile, pág. 139.

9. Santiago Sebastián, "La Huella ltaliana en la Arquitectura Colonial de Colombia y Ecuador", en "Boletín del Centro de Investigaciones Históricas y Estéticas", Caracas, Venezuela, Nov. 1971, NN 12.

10. E. Pereira Salas, "La Arquitectura Chilenaen el Siglo XIX", Ed. de los Anales de la U. de Chile, pág. 21.

11. Por gentileza de la Profesora Myriam Waisberg hemos visto el corte por la rotonda o hall de la casa de Dn. Francisco Ossa, del relevantamiento hecho por ella con un equipo de colaboradores, antes de su demolición en 1976.

12. Aristóteles Berlendis, "Perfil moral de Fermín Vivaceta", $1^{\text {er }}$ Centenario de la Sociedad de Artesanos "La Unión", pág. 6, 1962.

13. En "Vida y Obras del Arquitecto Don Fermín Vivaceta" de Arturo Blanco A., Santiago. 1924. Se lamenta don Arturo de don Vicente Grez "al escribir en francés su obra Les Beaux-Arts au Chili"... ni siquiera nombró a Vivaceta! Como si éste no hubiera existido. le hizo el vacío..."

A veces el silencio puede ser un arma de doble filo.

14. Primer Centenario de la Sociedad de Artesanos "La Unión". 1862-1962, pág. 48.

15. Arturo Blanco A. Op. cit. pág. 28.

Para la realización de este articulo he consultado, fuera de las obras citadas, varios seminarios inéditos, del Departamento de Historiay Teoria de la Arquitectura; entre otros: "Lalglesia y Convento de San Agustín "de Mario Luppi. "La Iglesia dela Veracruz" de Fernando Manterola. "Basilica y Convento de La Merced" de Luis Pachón H., "Iglesia y Convento de San Francisco* de W. Tapia

"La Catedral de Santiago" de Augusto Iglesias y Enrique Porte, ed. Instituto de Historia de la Arquitectura, Santiago, 1955

Las fotografias son del archivo del Laboratorio Central de Fotografia de la Universidad de Chile.

El dibujo del perfil de Vivaceta en la portada de la Revista "El Comercio" de abril de 1886 me ayudó a buscarlo Hernán Rodriguez V., Director del Museo Histórico Nacional, en el archivo del Museo.

Deseo agradecer las facilidades que me han otorgado los directivos de la Sociedad de Artesanos "La Unión", al permitirme consultar personalmente las magníficas obras de arquitectura de la biblioteca de Fermín Vivaceta. En especial, a su Presidente señor Armando Aguirre, al integrante del Directorio señor Domingo Sibila y al archivero señor Luis Navarrete. Sin su cooperación, este modesto trabajo no habría descubierto nada nuevo para la memoria de nuestro ilustre homenajeado.
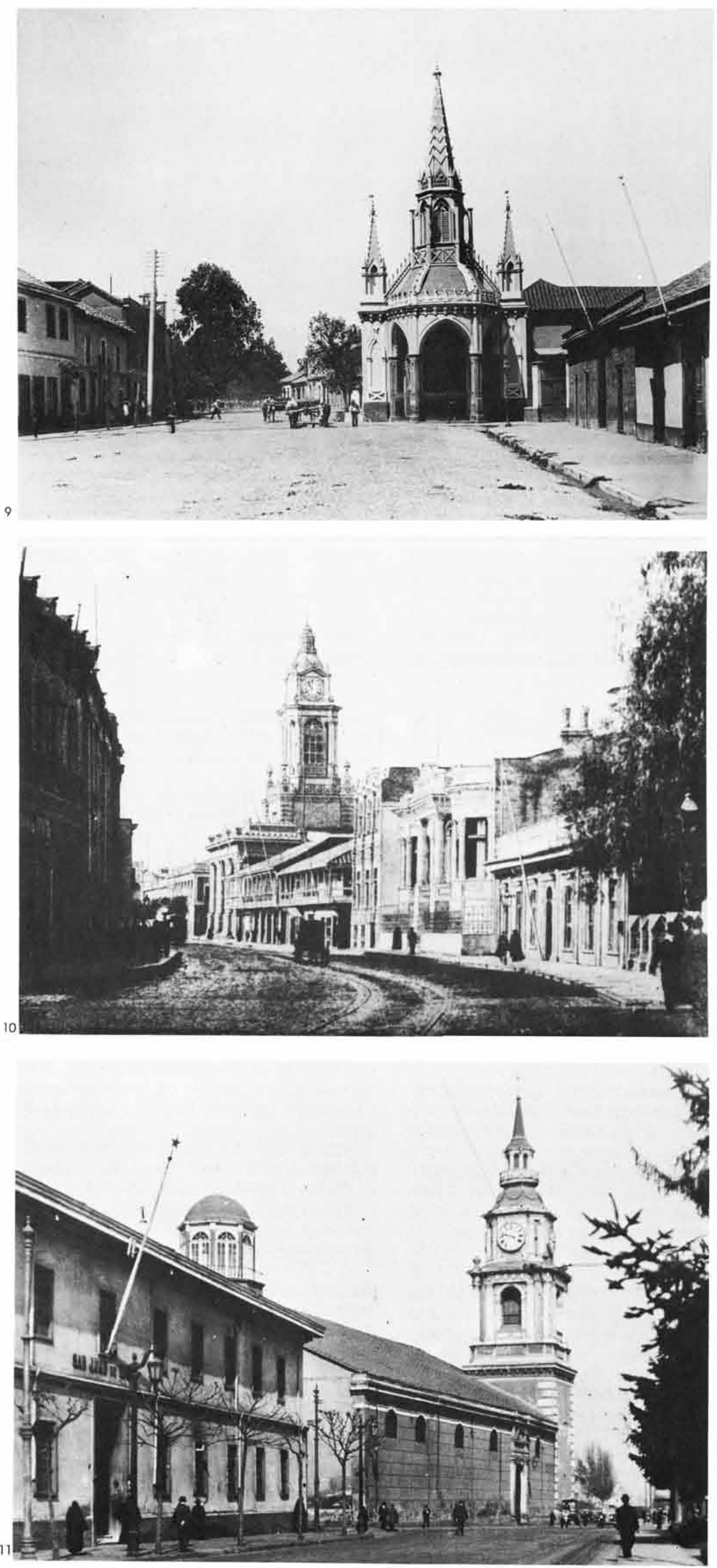